\title{
A influência do carboidrato na ativação cerebral durante exercício físico
}

\section{The carbohydrate influence on the brain activation during exercise}

\author{
Gabriela Kaiser Fullin Castanho', Eduardo Bodnariuc Fontes ${ }^{2}$, Brunno Machado de \\ Campos $^{3}$, João Guilherme Cren Chiminazzo ${ }^{1}$, Paula Teixeira Fernandes ${ }^{1 *}$
}

\begin{abstract}
$\mathrm{O}$ uso de carboidrato $(\mathrm{CHO})$ como suplemento nutricional está relacionado com melhor rendimento esportivo. Alguns estudos observaram relação entre consumo e ativação cerebral que influenciam o rendimento. Assim, o objetivo desse estudo foi avaliar a influência do consumo de CHO na ativação de determinadas áreas cerebrais durante exercício físico, realizado juntamente à aquisição de ressonância magnética funcional (RMf). Dez homens ciclistas $(32,1 \pm 4,1$ anos, peso 76,8 $\pm 14,6 \mathrm{~kg})$ realizaram um protocolo de exercício de pedalada, com alta intensidade (Escala de Borg), em cicloergômetro acoplado à ressonância magnética $(\mathrm{RM})$ e, ingeriram $50 \mathrm{~g}$ de $\mathrm{CHO}$ ou de placebo no intervalo de duas séries de exercício. A ingestão de $\mathrm{CHO}$ apresentou influência nas áreas cerebrais durante o exercício, ativando áreas relacionadas à tomada de decisões (insula) e motivação (sistema límbico) e desativando principalmente áreas motoras (lobo frontal) e de introspecção (precuneos). Com o consumo de placebo, também houve ativação de áreas importantes relacionadas à motivação do individuo (cíngulo posterior). Além disso, as áreas relacionadas com a iniciação e manutenção de movimento, localizadas no lobo frontal e cerebelo, apresentaram-se ativas. Com o $\mathrm{CHO}$, áreas importantes para a manutenção do exercício foram ativadas (BOLD positivo), mostrando que a suplementação pode ter influência na ativação cerebral durante o exercício de forma que melhore o rendimento esportivo.
\end{abstract}

Palavras-chave: neurociências do esporte, carboidrato, exercício físico, ressonância magnética funcional, cérebro.

\begin{abstract}
The use of carbohydrate $(\mathrm{CH})$ as a nutritional supplement is related to better sports performance. Some studies have noted a relationship between consumption and brain activation influencing the performance. The objective of this study was to evaluate the influence of $\mathrm{CH}$ consumption in the activation of certain brain areas during exercise, performed simultaneously the acquisition of functional magnetic resonance imaging (fMRI). Ten men cyclists $(32.1 \pm 4.1$ years, weight $76.8 \pm 14.6 \mathrm{~kg})$ performed a pedaling exercise protocol, with high intensity (Borg Scale), on a cycleergometer coupled to magnetic resonance (MR) and ingested $50 \mathrm{~g} \mathrm{CH}$ or placebo in the range of two sets of exercise. The $\mathrm{CH}$ ingestion showed influence on brain areas during exercise, activating areas related to decision-making (insula) and motivation (limbic system) and mainly disabling motor areas (frontal lobe) and introspection (precuneus). With the use of placebo, there was also activation of important areas in the motivation of the individual (posterior cingulate). In addition, areas associated with the initiation and maintenance of movement, located on the front lobe and cerebellum, was active. With the use of $\mathrm{CH}$, areas important for maintenance of the exercise have been activated showing that supplementation can influence the brain activation during exercise to improve the sport performance.

Keywords: sports neurosciences, carbohydrate, exercise, functional magnetic resonance, brain.
\end{abstract}

\footnotetext{
Artigo recebido a 08.03.2015; Aceite a 25.09.2015

${ }^{1}$ Faculdade de Educação Física, Universidade Estadual de Campinas, UNICAMP, Campinas, Brasil

${ }^{2}$ Universidade Federal do Rio Grande do Norte, Natal, Brasil

${ }^{3}$ Faculdade de Ciências Médicas, Universidade Estadual de Campinas, UNICAMP, Campinas, Brasil

* Autor correspondente: Av. Érico Veríssimo, 701, Cidade Universitária "Zeferino Vaz" Barão Geraldo, Campinas - SP, Brasil, CEP: 13.083-851 E-mail: paula@fef.unicamp.br
} 


\section{INTRODUÇÃO}

A nutrição adequada tem sido relacionada ao melhor desempenho esportivo, sendo uma dieta variada em carboidrato $(\mathrm{CHO})$, proteína, gordura e micronutrientes, importante para manutenção da saúde e para melhora do desempenho. Para isso, alguns autores investigaram qual seria o melhor substrato para a prática de exercício físico e o $\mathrm{CHO}$ foi apontado como um dos mais promissores para essa finalidade (Anantaraman, Carmines, Gaesser, \& Weltman, 1995; Burke, Hawley, Wong, \& Jeukendrup, 2011; Carter, Jeukendrup, \& Jones, 2005; Carter, Jeukendrup, Mundel, \& Jones, 2003).

Tem sido demonstrada a importância do consumo de $\mathrm{CHO}$ no rendimento desportivo, pois quando os estoques de $\mathrm{CHO}$ estão baixos e ocorre a baixa disponibilidade do mesmo para o organismo, o desempenho é afetado negativamente (Jeukendrup, 2003, 2004). Assim, o consumo de $\mathrm{CHO}$ pode ajudar no exercício através dos seguintes mecanismos: manutenção da glicemia, preservação de glicogênio hepático e atraso ou atenuação da fadiga central (Jeukendrup, 2004).

Alguns estudos recentes (Chambers, Bridge, \& Jones, 2009; Fares \& Kayser, 2011; Gam, Guelfi, \& Fournier, 2013) têm sugerido que enxaguantes bucais com $\mathrm{CHO}$ na composição podem contribuir para o melhor rendimento, isso porque quando esses enxaguantes entram em contato com a mucosa da boca, a mesma tem receptores bucais que enviam informações ao cérebro de que o $\mathrm{CHO}$ (presente no enxaguante) estaria sendo consumido (Chambers et al., 2009). O cérebro é metabolicamente ativo e, durante o exercício físico intenso, as exigências centrais de energia podem ser mais difíceis de sustentar por causa do aumento na demanda de energia periférica (Ide \& Secher, 2000). Sabe-se que a glicose é a principal energia para o cérebro em condições normais fisiológicas e ambientais (Evans \& Amiel, 1998), levantando-se a hipótese de que o consumo de $\mathrm{CHO}$ poderá ajudar o cérebro a se manter em perfeito funcionamento durante o exercício.

Áreas cerebrais que estejam com maior atividade e maior fluxo sanguíneo demandam maior transporte de glicose e oxigênio a fim de suprir as necessidades energéticas de suas células nervosas assim, o consumo de $\mathrm{CHO}$ ajuda a aumentar suas concentrações de glicose (Buxton, 2000). No entanto, pouco se sabe sobre a ativação das áreas cerebrais durante o exercício físico e se o consumo de $\mathrm{CHO}$ pode afetar essa ativação.

Estudos de neuroimagem (Chambers et al., 2009; Gant, Stinear, \& Byblow, 2010) têm demonstrado que a ingestão de glicose produz ativação de áreas cerebrais que podem estar relacionadas a respostas comportamentais e autonômicas. Um dos métodos mais conhecidos para avaliar o funcionamento das áreas cerebrais é a Ressonância magnética funcional (RMf), pois é capaz de avaliar alterações do estado funcional do cérebro de forma não invasiva, incluindo regiões profundas, com alta resolução espacial, sem uso de contrastes intravenosos e radiação ionizante (Heeger \& Ress, 2002). Assim, é possível descobrir quais áreas específicas do cérebro estão ativas através de alterações hemodinâmicas, que se baseiam em flutuações de sinal BOLD (Blood Oxygen Level Dependent) ou sinal dependente do nível de oxigênio no sangue, causada pelo aumento na atividade neural (Buxton, 2000).

A maioria dos estudos existentes com utilização de RMf são restritos a pequenos grupos musculares como músculos do braço avaliados durante preensão manual, pois a movimentação da cabeça deve ser a mínima dentro da RMf (Benwell, Mastaglia, \& Thickbroom, 2007; Dai, Liu, Sahgal, Brown, \& Yue, 2001; Liu et al., 2007). Por isso, exercícios que envolvem grandes grupos musculares, como por exemplo, o ciclismo, parece ser mais atrativo para investigar o papel do cérebro na regulação do exercício, já que esta forma de exercício é conhecida por estimular grande parte do organismo, observando-se diversas respostas fisiológicas (Calbet, Radegran, Boushel, \& Saltin, 2009; Franke, Boettger, \& McLean, 2000) que podem refletir em atividades cerebrais também complexas.

Neste sentido, para estudar o funcionamento do cérebro durante o exercício, é necessário avaliá-lo no momento que o exercício está sendo realizado. Assim, optou-se por formular um 
cicloergômetro compatível com ressonância magnética (RM), e através de aquisições de RMf realizadas de forma concomitante com o mesmo, o presente estudo teve por objetivo avaliar o efeito da ingestão de $\mathrm{CHO}$ na atividade cerebral durante exercício físico de pedalar.

\section{Amostra}

\section{MÉTODO}

Foram selecionados dez homens $(32.1 \pm 4.1$ anos) com pelo menos 6 meses de experiência em ciclismo e que praticavam no mínimo 120 minutos por semana de exercício físico. Todos os participantes foram informados dos objetivos e procedimentos a que seriam submetidos e assinaram um Termo de Consentimento Livre e Esclarecido. O estudo foi aprovado pelo Comitê de Ética em Pesquisa da UNICAMP, sob parecer $n^{\circ} 766 / 2010$ e concluído em conformidade com a Declaração de Helsinki. Os participantes foram orientados a não consumir álcool e substâncias cafeinadas e a evitar atividade física extenuante nas 24 horas precedentes aos testes. Os critérios de exclusão do estudo foram: presença de sintomas de claustrofobia; presença de qualquer parafuso, prótese ou qualquer outro material magnético no corpo dos participantes; presença de doenças crônicas como diabetes, hipertensão e câncer, doenças neurológicas e psiquiátricas.

Todos os sujeitos responderam a um questionário com dados pessoais como idade, tabagismo, etilismo, consumo de drogas, escolaridade, histórico clínico e de doenças, uso de medicamentos e/ou suplemento e frequência de exercício físico.

\section{Delineamento do estudo}

Cada participante visitou o laboratório de Neuroimagem do Hospital das Clínicas da Universidade Estadual de Campinas (UNICAMP) em três ocasiões separadas com intervalo de no mínimo sete dias (conforme explicado no item visitas). Realizaram os testes em cicloergômetro acoplado à RM e consumiram 2 tipos de bebidas diferentes ( $\mathrm{CHO}$ e placebo).

\section{Instrumentos}

Cicloergômetro adaptado para o equipamento de RM

Para simulação do exercício de ciclismo, o participante realizou o protocolo de exercício no cicloergômetro adaptado para o equipamento de $\mathrm{RM}$, no qual pedalou em decúbito dorsal. Esse cicloergômetro foi construído recentemente, como um sistema para pedalar (na posição supina) que pode ser posicionado no interior da sala do equipamento de RM, e que transmite por meio de um eixo cardam, o torque que traciona o pé-de-vela do ciclossimulador (Computrainer, Racemate, Inc., USA) localizado na sala de comando do equipamento, juntamente ao computador onde são armazenadas as imagens para posterior análise (Fontes et al., 2015).

\section{Ressonância Magnética funcional (RMf)}

Os voluntários foram submetidos à aquisição de RMf (3T Achieva MR scanner, Philips Medical Systems, Best, The Netherlands) no Hospital das Clínicas da Faculdade de Ciências Médicas da UNICAMP. O protocolo para aquisição de imagens consistia em 2 EPIs (echo-planar image) com 5 minutos cada (tamanho do voxel de $3 \times 3 \times 3 \mathrm{~mm}^{3}, 40$ cortes axiais, sem gap, FOV $240 \times 240 \times 117 \mathrm{~mm}^{3}$, TE $30 \mathrm{~ms}$, TR 2000ms, ângulo de flip de $90^{\circ}$ ). Uma imagem anatômica T1 (180 cortes sagitais, sem gap, com tamanho do voxel de $1 \times 1 \times 1 \mathrm{~mm}^{3}$, FOV $240 \times 240 \times 180 \mathrm{~mm}^{3}$, TE 6.9 $\mathrm{ms}$, TR $3.1 \mathrm{~ms}$, ângulo de flip de $8^{\circ}$ ) foi usada para corregistro com as imagens funcionais.

\section{Substâncias a serem consumidas}

As substâncias consumidas foram: $\mathrm{CHO}=$ solução de carboidrato com $50 \mathrm{~g}$ de maltodextrina misturada a $300 \mathrm{ml}$ de água (concentração de $16,5 \%)$ quantidade selecionada pela recomendação do Colégio Americano de Medicina Esportiva (Rodriguez, Di Marco, \& Langley, 2009), porém com uma concentração maior devido ao desconforto de tomar muito líquido e ficar em posição de decúbito dorsal para pedalar; Placebo = bebida líquida feita com água e suco em pó dietético de sabor, cor e textura semelhantes à maltodextrina para que os indivíduos não soubessem quais substâncias estariam sendo ingeridas. 


\section{Procedimentos}

Visitas

$1^{\text {a }}$ Visita: Os participantes familiarizaram-se ao equipamento, aos procedimentos que seriam submetidos e realizaram teste incremental intermitente no ciclo ergômetro acoplado à RM. O protocolo do $1^{\circ}$ teste foi composto por blocos de 30 segundos de pedalada alternados com 30s de descanso, durante os quais os sujeitos eram instruídos a manter uma cadência de $60 \mathrm{rpm}$ (rotações por minuto) (Figura 1).

A escala de Borg (Borg, 1962), com pontuação de 6 a 20, estava constantemente disponível em uma tela interna do equipamento de RM. Durante o experimento, os participantes tinham que reportar a perceção subjetiva de esforço (PSE) com as mãos após completar cada período de pedalada. O teste começou com carga de 50W, e tinha incremento de $25 \mathrm{~W}$ a cada 4 blocos de exercício (Figura 1). Esse teste inicial foi utilizado para definir a carga a ser trabalhada nos testes da $2^{\circ}$ e $3^{\circ}$ visita, que corresponderia ao número 15 da escala de Borg (PSE INTENSO) para o indivíduo.

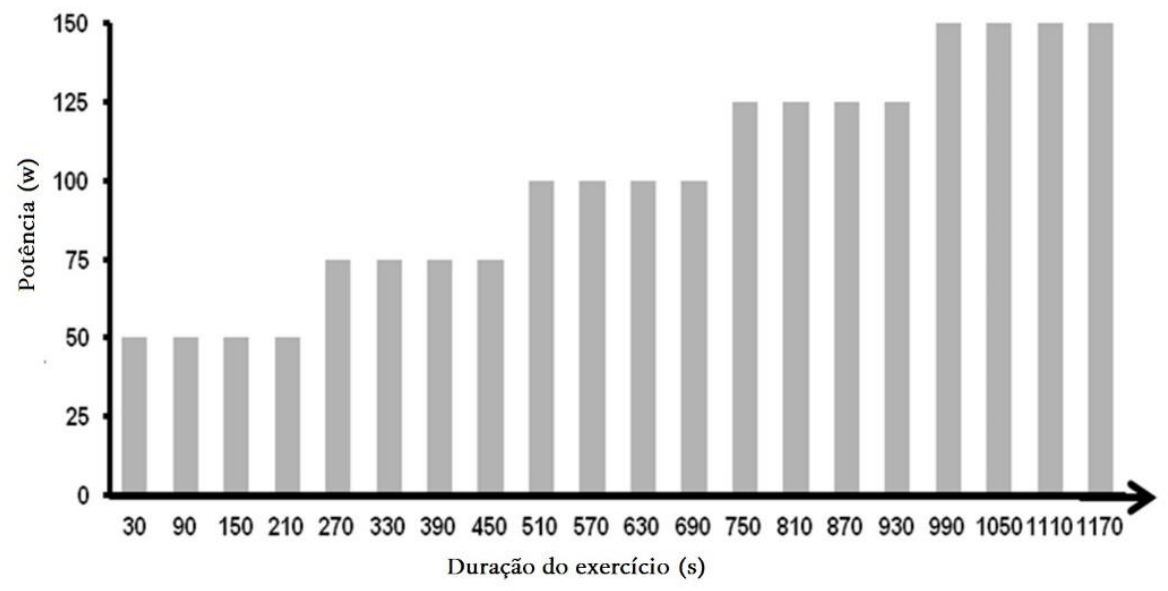

Figura 1. Protocolo de exercício com carga incremental, com a aquisição concomitante de RMf.

$2^{\mathrm{a}}$ e $3^{\mathrm{a}}$ Visitas: Os participantes foram orientados (pela pesquisadora) a comparecerem seguindo a dieta habitual nos dias das $2^{\circ}$ e $3^{\circ}$ visitas, não consumirem suplementos alimentares 2 dias antes dos testes e estarem em jejum de 6 horas para a hora marcada para os testes. Todos os participantes consumiram $\mathrm{CHO}$ e placebo, de forma aleatória, sem terem o conhecimento de qual bebida estavam ingerindo, caracterizando um cross-over. Se o indivíduo consumisse $\mathrm{CHO}$ na $2^{\text {a }}$ visita, ele consumiria o placebo na $3^{\mathrm{a}}$ e vice-versa. Apenas a pesquisadora sabia quais substâncias os participantes consumiam.

O protocolo dos testes da $2^{\circ}$ e $3^{\circ}$ visitas realizadas consistiu em blocos de exercício no ciclo ergômetro com a carga estipulada (em Watts) para cada indivíduo como sendo "intenso" (número 15) de acordo com a escala de PSE de Borg realizada no primeiro dia de teste. O indivíduo realizava 2 minutos iniciais de aquecimento, depois pedalava 30 segundos, alternados com 30 segundos de descanso, foram 4 blocos de descanso e pedalada, sendo que após os blocos de pedalada o indivíduo reportava a PSE com as mãos. Pausou-se a aquisição da RMf por 5 minutos para que o individuo saísse da RM e consumisse a bebida e depois voltou para dentro da RM e o protocolo de exercício e os mesmos blocos foram repetidos (Figura 2).



Figura 2. Protocolo de exercício com a ingestão de carboidrato ou placebo entre as aquisições concomitantes à RMf. 


\section{Análise Estatística}

A análise estatística para caracterização da amostra foi realizada no Microsoft Excel versão 2010 através de média e desvio-padrão e teste-T de Student para verificar diferença estatística entre a PSE dos participantes em cada condição (consumo de substâncias), com $\mathrm{p}<0.05$.

As análises de RM foram divididas em dois níveis estatísticos. No primeiro nível (individual), o paradigma em bloco realizado pelo ciclista durante a RM (Figura 4) foi utilizado na elaboração de um GLM (Modelo Linear Geral). Com esta metodologia buscou-se as respostas BOLD relacionadas a cada condição (atividade ciclística) separadamente, sob as diferentes condições aplicadas. Para cada condição, um mapa de contraste estatístico foi criado e representava espacialmente o grau de sincronia das oscilações do BOLD com o paradigma experimental aplicado. $\mathrm{Na}$ análise de segundo nível, foi realizado teste-T de Student para uma amostra ( $p<0.001$, não corrigido), a fim de se verificar as regiões estatisticamente significativas que eram coincidentes sob as diferentes condições ( $\mathrm{CHO}$ e placebo), as imagens construídas com esses testes foram suavizadas antes do teste ser aplicado.

O pré-processamento na RMf e as análises estatísticas subsequentes foram realizadas no Statistical Parametric Mapping (SPM8, http://www.fil.ion.ucl.ac.uk/spm/) como ferramenta para plataforma Matlab 7.7 (The MathWorks, Natick, USA). No processo de préprocessamento das imagens, elas eram realinhadas devido ao movimento do paciente, normalizados para o padrão MNI (Montreal Neurological Institute 152), e suavizadas com um filtro gausiano tridimensional com kernel de $6 \mathrm{~mm}^{3}$ (FWHM), para reduzir variações ruidosas de sinal entre voxels vizinhos.

\section{RESULTADOS}

Neste estudo foram avaliados dez homens, cujas principais características foram $32.1 \pm 4.1$ anos, $76.8 \pm 14.6 \mathrm{~kg}$, estatura média de $1.74 \pm$ $0.08 \mathrm{~m}$ e média de $480 \pm 230$ minutos de exercício físico na semana.

A PSE foi avaliada nos momentos após os blocos de pedalada e feita média dos quatro momentos antes do consumo da bebida e após o consumo de bebida. Quando se avaliou a diferença entre o consumo de placebo e carboidrato, não houve diferença significativa $(p=0.17)$. Também não houve diferença quando comparado antes e após o consumo, independente da substância $(p=0.43$ com placebo e $p=0.94$ com $\mathrm{CHO}$ ).

Verificou-se que o $\mathrm{CHO}$ ativou áreas do lobo frontal e parietal, cerebelo além da insula, hipocampo e giro do cíngulo, esses últimos sendo do sistema límbico. Realizou-se uma comparação não estatística das áreas que o $\mathrm{CHO}$ ativou de diferente do placebo e pôde-se observar que essas compreenderam lóbulo paracentral, giro parahipocampal, lóbulo parietal, insula e giro do cíngulo. O placebo ativou áreas dos lobos frontal, temporal, parietal e límbico (cíngulo posterior) e também o núcleo caudado, sendo que esse e o subgiro não se mostraram ativos com o consumo de CHO. A diminuição da ativação (BOLD negativo) se mostrou muito semelhante com as duas substâncias, sendo que o placebo também desativou o giro hipocampal e o lingual, que não sofreram a mesma reação com o consumo de CHO (tabela 1).

Para avaliar o efeito de se consumir o $\mathrm{CHO}$ ou o placebo, independente de qual, foram analisadas as ativações de antes do consumo das duas substâncias com após o consumo. Os resultados mostraram que o ato de consumir a substância tem diferença na ativação das áreas. Antes do consumo, áreas como cerebelo, tálamo e núcleo lentiforme estavam ativas, porém, após o consumo, as mesmas não se mostraram mais ativas. Além disso, com o BOLD negativo, observou-se desativação do giro fusiforme, uncus e precuneos após o consumo das substâncias (tabela 2). 
Tabela 1

Áreas cerebrais com respostas ao BOLD comuns com o consumo de cada substância.

\begin{tabular}{lll}
\hline & \multicolumn{1}{c}{ BOLD POSITIVO } & \multicolumn{1}{c}{ BOLD NEGATIVO } \\
\hline & Lóbulo paracentral E & Giro occiptal médio E/DGiro \\
& Giro pós-central ECulmen E/D frontal superior E/D \\
& Giro parahipocampal E & Giro frontal médio E/D \\
Áreas comuns com o consumo de & Lóbulo parietal inferior E/D & Giro temporal inferior D \\
carboidrato & Insula E & Giro occipital superior D \\
& Giro do cíngulo E/D & Uncus E \\
& Giro pré-central E & Cuneos D \\
& & Giro temporal superior D \\
\hline & Giro pós-central E & Giro frontal superior E/D \\
& Giro pré-central D & Giro temporal superior E/D \\
& Culmen D & Cuneus D \\
Cíngulo posterior E & Giro frontal inferior D \\
& Giro fusiforme E & Giro occiptal médio E \\
& Giro frontal médio E & Giro parahipocampal D \\
& Caudado E & Giro lingual D \\
& Subgiro E & Giro frontal médio E \\
\hline
\end{tabular}

BOLD: Blood Oxigen Level-Dependent, E: esquerdo; D: direito.

Tabela 2

Áreas cerebrais com respostas ao BOLD antes e após o consumo das substâncias.

\begin{tabular}{lll}
\hline & \multicolumn{1}{c}{ BOLD POSITIVO } & \multicolumn{1}{c}{ BOLD NEGATIVO } \\
\hline & Lóbulo paracentral E & Giro frontal médio E/D \\
& Giro pós-central E & Giro frontal superior E/D \\
& Declive cerebelo D & Cuneos D \\
Lóbulo parietal inferior D & Giro occipital médio E/D \\
Giro & Giro temporal médio E \\
& Gálamo D & Giro temporal superior E \\
& Núcleo lentiforme E & Giro frontal inferior D \\
& Giro pós-central E/D & Giro frontal superior E/D \\
& Lóbulo parietal inferior D & Giro frontal médio E \\
& & Giro temporal superior E/D \\
Após o consumo das substâncias & Uncus E/D \\
& & Giro fusiforme D \\
& & Cuneos D \\
& & Giro occipital médio E/D \\
& Giro temporal médio E \\
& & Giro frontal inferior D \\
& & Precuneos D \\
\hline
\end{tabular}

BOLD: Blood Oxigen Level-Dependent, E: esquerdo; D: direito.

As figuras 3 a 10 abaixo são os pontos que apresentaram maior magnitude do sinal BOLD positivo ou negativo em relação a cada situação, conforme apresentado na tabela 2 . A figura 3 mostra claramente o córtex motor (lobo frontal), cerebelo e insula ativos com o consumo de $\mathrm{CHO}$, o lobo frontal e cerebelo também aparecem na figura 4, relacionada ao consumo de placebo, além do lobo parietal e temporal do lado esquerdo.

A figura 5 mostra as áreas ativas antes do consumo das substâncias. Observou-se que áreas do lobo frontal, temporal e parietal, sublobo e cerebelo estavam ativas, o que não se viu na figura 6, que ilustra as áreas ativas após o consumo, lobo parietal e frontal.

A diminuição da ativação (BOLD negativo) teve poucas diferenças com o consumo das substâncias (tabela 1), mas as imagens das desativações diferiram entre si, sendo que na figura 7 observou-se a desativação de áreas occipitais com o consumo de $\mathrm{CHO}$ e, o placebo desativou áreas do lobo frontal (figura 8). 

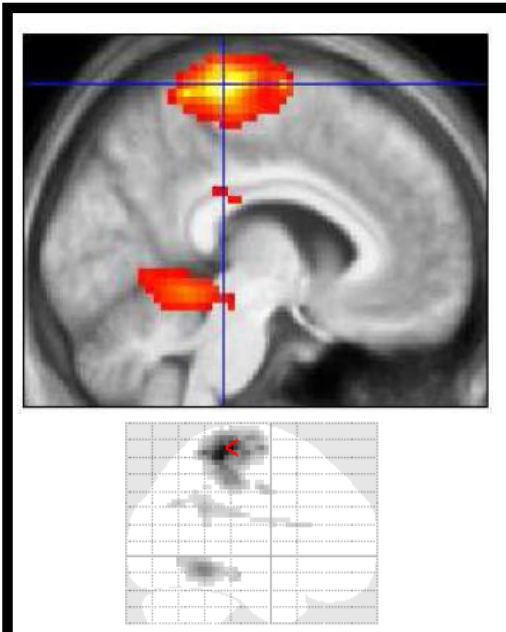
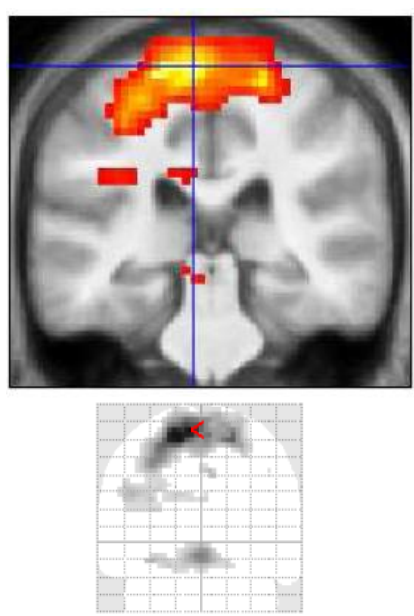
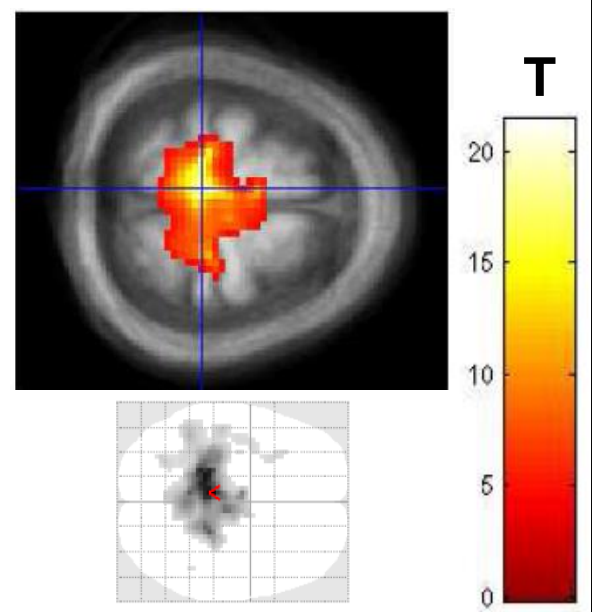

Figura 3. Mapa de BOLD positivo $(p<0.001$ tamanho cluster $=0)$ relativo a áreas comuns com o consumo de carboidrato. BOLD: Blood Oxigen Level-Dependent.



Figura 4. Mapa de BOLD positivo $(p<0.001$ tamanho cluster $=0$ ) relativo a áreas comuns com o consumo de placebo. BOLD: Blood Oxigen Level-Dependent.

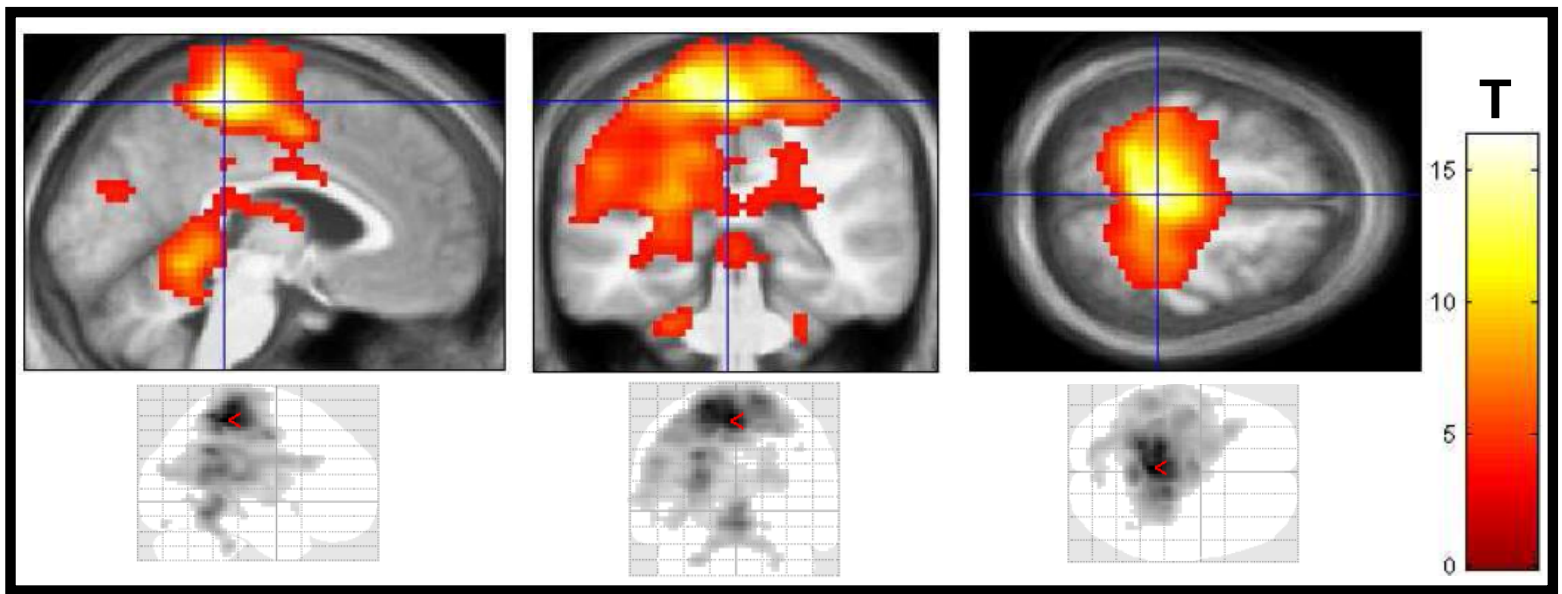

Figura 5. Mapa de BOLD positivo ( $p<0.001$ tamanho cluster $=0)$ relativo a áreas comuns, anterior ao consumo de substâncias. BOLD: Blood Oxigen Level-Dependent. 




Figura 6. Mapa de BOLD positivo $(p<0.001$ tamanho cluster $=0)$ relativo a áreas comuns, após o consumo de substâncias. BOLD: Blood Oxigen Level-Dependent.



Figura 7. Mapa de BOLD negativo $(p<0.001$ tamanho cluster $=0)$ relativo a áreas comuns com o consumo de carboidrato. BOLD: Blood Oxigen Level-Dependent.

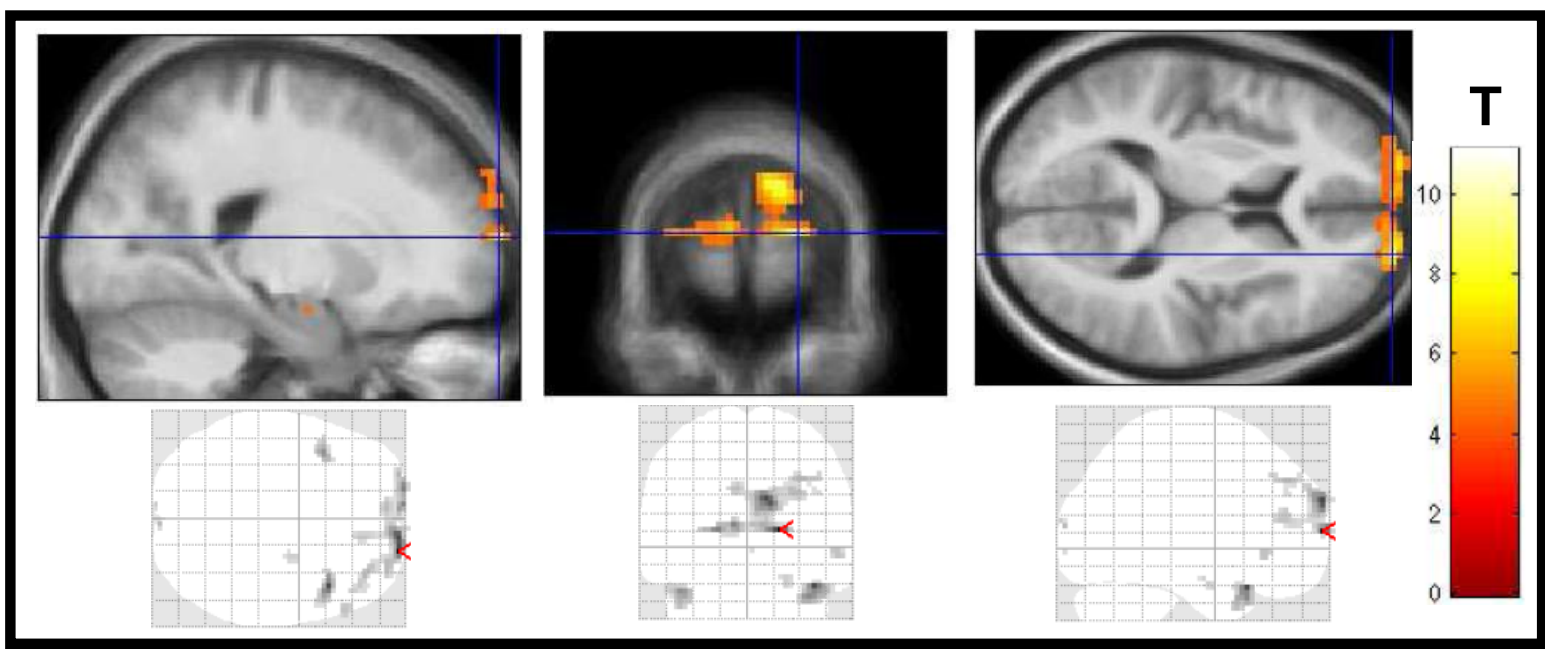

Figura 8. Mapa de BOLD negativo $(p<0.001$ tamanho cluster $=0)$ relativo a áreas comuns com o consumo de placebo. BOLD: Blood Oxigen Level-Dependent. 
As imagens relativas aos momentos antes do consumo das duas substâncias mostraram desativação de diversas aéreas do lobo frontal, occipital, temporal e cíngulo anterior (figura 9).
Quando houve o consumo de substância, a desativação se manteve na maioria das áreas apenas não aparecendo mais o cíngulo anterior, de acordo com a figura 10.

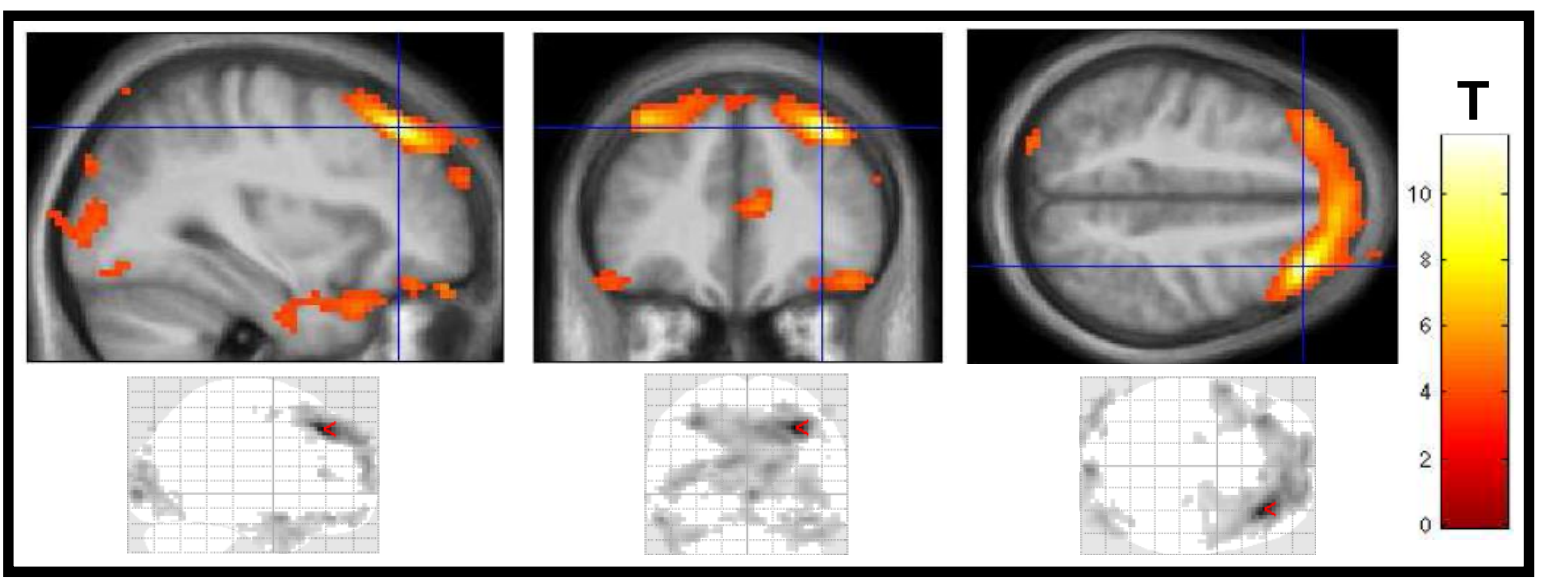

Figura 9. Mapa de BOLD negativo ( $p<0.001$ tamanho cluster $=0$ ) relativo a áreas comuns, anterior ao consumo de substâncias. BOLD: Blood Oxigen Level-Dependent.

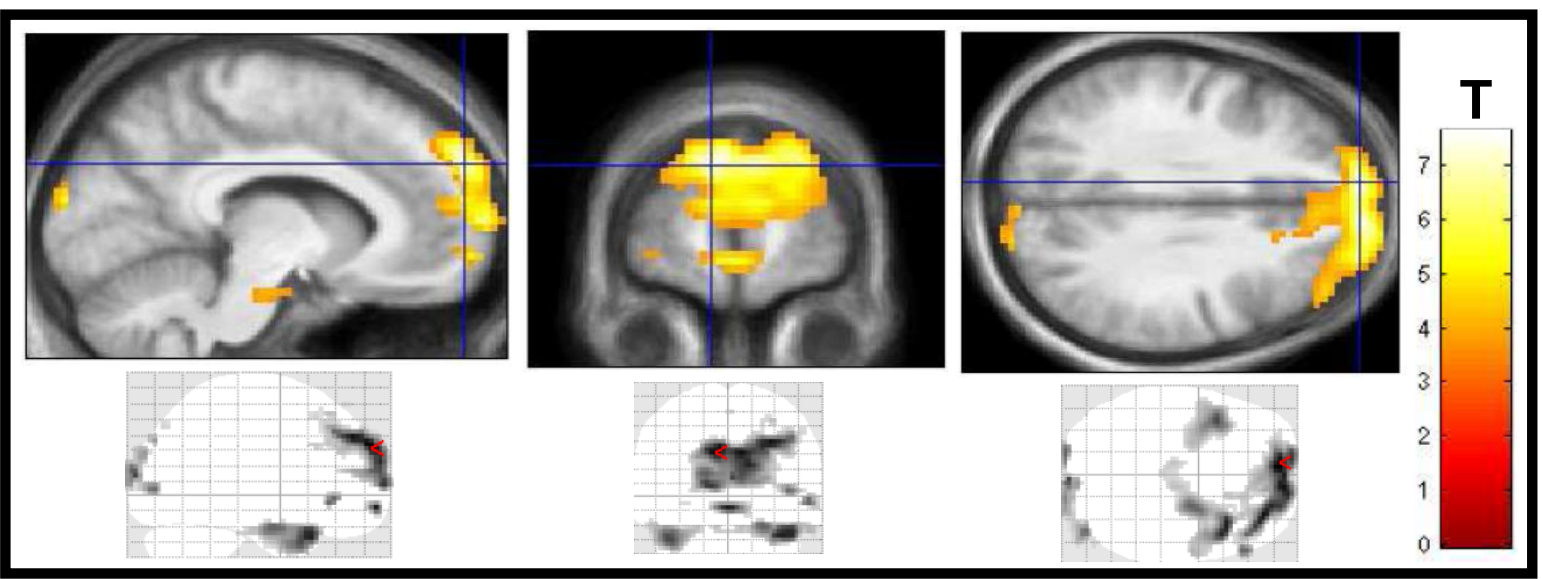

Figura 10. Mapa de BOLD negativo $(p<0.001$ tamanho cluster $=0)$ relativo a áreas comuns, após o consumo de substâncias. BOLD: Blood Oxigen Level-Dependent.

\section{DISCUSSÃo}

Através dos resultados de ativação observados nas análises para uma amostra, os principais achados foram que a ingestão de $\mathrm{CHO}$ ativou (BOLD positivo) o lóbulo paracentral, o giro parahipocampal, o lóbulo parietal e a insula, áreas que o placebo não ativou. O placebo ativou giro pós-central, córtex do cíngulo anterior (CCA) e posterior, núcleo caudado, giro fusiforme, precuneos, cuneos. O giro pós-central localizado no lobo parietal está relacionado à área somatossensorial. Estudos (Christensen et al., 2000; Mehta, Verber, Wieser, Schmit, \& Schindler-Ivens, 2009) mostraram que o aumento na ativação do córtex motor primário, pré-motor, córtex somatossensorial, área suplementar e cerebelo está relacionado à execução, controle e coordenação de movimentos. O cíngulo posterior está relacionado a estímulos nociceptivos, como a dor (Bromm, 2001) e com raiva (Park et al., 2010), enquanto o núcleo caudado está associado a emoções positivas, como felicidade e amor (Bartels \& Zeki, 2000).

As duas substâncias ativaram áreas do córtex frontal, temporal e cerebelo. O córtex motor primário (giro pré-central) e o cerebelo se apresentaram ativos com o consumo das duas substâncias, sendo que ambos foram associados a tarefas motoras e coordenadas em estudos que envolviam menores membros (Sidhu, Hoffman, Cresswell, \& Carroll, 2012; Thach, 1998). Esses 
achados confirmam a contribuição destas áreas para o controle da atividade motora durante o exercício.

Davis e Brown (2001) sugeriram que o consumo de $\mathrm{CHO}$ durante o exercício ajuda a reduzir a concentração de adrenalina, glucagon, cortisol e hormônio do crescimento no sangue e a aumentar a concentração da insulina. Estes fatores podem retardar a depleção de glicogênio muscular e hepático, aumentando a captação de glicose e a oxidação nos músculos e cérebro. Além dos benefícios físicos, o $\mathrm{CHO}$ também mostrou ter efeitos no estado psicológico do atleta, incluindo redução na sensação de esforço, aumento da motivação, bom humor e diminuição da inibição do centro nervoso associado à atividade motora (córtex motor), situado na região superior do cérebro no lobo frontal. Adicionalmente, o $\mathrm{CHO}$ contribui na diminuição da concentração de ácidos graxos livres e amônia, substancias que colaboram com o início da fadiga central.

Além do córtex motor e pré-motor, motora suplementar, o lobo frontal inclui o córtex préfrontal, que está associado com processamento de informações (Miller, Freedman, \& Wallis, 2002) e pode ser essencial para a modulação da função cognitiva durante o exercício. Além disso, a porção dorsolateral do córtex pré-frontal está associada com a consciência auto-reflexiva (Vogeley, Kurthen, Falkai, \& Maier, 1999).

Os resultados do presente estudo corroboraram com outros estudos, pois Fontes et al. (2015) verificaram que pedalar no cicloergômetro dentro da RM ativou áreas no giro pré-central (córtex motor primário), giro póscentral (córtex somatossensorial primário) e cerebelo durante o exercício, quando comparado aos momentos de descanso. O que também ocorreu nos achados de Mehta, Verber, Wieser, Schmit, e Schindler-Ivens (2009) que avaliaram a função cerebral associada ao ciclismo e verificaram um aumento de sinal BOLD durante exercício no córtex motor primário e cerebelo, comparado ao descanso, porém a intensidade individual não foi ajustada e a RMf foi realizada após o término do exercício.

Hilty, Langer, Pascual-Marqui, Boutellier, e Lutz (2011) avaliaram 16 homens saudáveis pedalando a $65 \%$ VO2pico até exaustão e acessaram a ativação cerebral através de EEG, os resultados mostraram ativação do córtex parietal e áreas límbicas após teste incremental de ciclismo, associando-os à fadiga muscular e também ativação do córtex orbitofrontal medial.

$\mathrm{O} \mathrm{CHO}$ e o placebo desativaram (BOLD negativo) áreas também do lobo frontal e temporal, além do lobo occipital. Entretanto o CHO desativou uma área do sistema límbico, o uncus, e o placebo desativou outra área do mesmo sistema, o giro parahipocampal, apresentando assim uma diferença entre as duas substâncias. O lobo límbico é o circuito básico de emoções funcionalmente ligado à motivação, emoções e memória (McLachlan, 2009), mostrando ser uma área importante para as decisões durante o exercício.

Quando se somou os momentos pré consumo e pós consumo das duas substâncias, buscou-se avaliar a influência de consumir algo durante o exercício, independente da substância. Neste estudo observou-se que antes do consumo muitas áreas estavam ativas e que após a ingestão de substância este padrão alterou-se sendo que apenas duas áreas do lobo parietal eram coincidentes para o $\mathrm{CHO}$ e o placebo.

Outro estudo com ciclismo mostrou ativação o lobo parietal e sistema límbico após teste incremental de ciclismo, associando-os à fadiga muscular (Schneider, Brummer, Abel, Askew, \& Struder, 2009). Isso mostra que com a prática de qualquer exercício pode ocorrer a mudança psicológica individual.

Em consonância com os resultados apresentados, um estudo realizado com ratos verificou que durante o exercício se ativavam o córtex motor e o cerebelo; áreas sensoriais (somatossensorial, córtex visual, sub-tálamo, tálamo) e de controlo autonômico como hipotálamo, hipocampo e amígdala (Vissing, Andersen, \& Diemer, 1996).

A desativação ocorreu em diversas áreas, tanto antes quanto após o consumo, sendo a maioria do lobo frontal, temporal e parietal. O $\mathrm{CHO}$ e o placebo desativaram também o sistema límbico (uncus) e o precuneos. $\mathrm{O} \mathrm{CHO}$ apresentou desativação do precuneos, quando comparado ao controle (antes do consumo das 
substâncias) e, áreas do lobo frontal quando comparado ao placebo. O precuneos foi associado com características comportamentais e cognitivas associadas à introspecção (Cavanna, 2007).

Como apresentado, o $\mathrm{CHO}$ mostrou ativação na insula e CCA (comparado ao controle), a insula é uma área relacionada à tomada de decisão (Craig, 2009), o que pode ser crucial para a decisão de parar ou não o exercício. Juntamente com o CCA, tem um papel importante nos mecanismos de conscientização dos fatores fisiológicos envolvidos no exercício dinâmico (dor crônica e as diferenças de temperatura térmica, tensão muscular) e suas respostas a esses estímulos, alterando a motivação e as emoções do indivíduo com o exercício (Craig, 2002, 2009). Também podem regular a homeostase para respostas conscientes, afetivas e emocionais, durante o exercício por respostas finas para a PSE. Porém, o envolvimento dessas áreas no exercício tem sido exaustivamente estudado (Craig, 2009; Medford \& Critchley, 2010).

Além disso, estudos relacionaram o aumento na atividade neural do CCA e insula com dor muscular induzida (Henderson, Bandler, Gandevia, \& Macefield, 2006; Kupers, Svensson, \& Jensen, 2004), o que pode ter relação com a ativação das mesmas no presente estudo, pois os testes foram feitos em posição diferente do habitual dos atletas, podendo ser desconfortável. A insula também apareceu ativa em estudo de Williamson, McColl, Mathews, Ginsburg, e Mitchell (1999) com a alta intensidade de exercício e, em estudo de Chambers, Bridge, e Jones (2009), que ofereceram enxaguante bucal com glicose e com adoçante para os atletas e encontraram ativação da ínsula e também a área do CCA com a glicose, assim como nos resultados apresentados acima. Porém o consumo de adoçante, utilizado como placebo, também ativou a insula.

No presente estudo, o placebo ativou o córtex do cíngulo posterior, sendo que essa área foi descrita como uma área integradora entre o sensorial, motor, visceral, motivacional e emocional (Vogt, 2009) e no qual a região posterior é ativada principalmente durante divagação mental (Mason et al., 2007) ou quando estímulos emocionais tem um significado pessoal (Maddock, Garrett, \& Buonocore, 2001). Além disso, a região posterior está relacionada na avaliação dos eventos e comportamentos sensoriais ligados ao movimento e à memória (Vogt, Finch, \& Olson, 1992), atuando como um pré-processamento emocional, para que a informação emocional possa alcançar outras subregiões, como o córtex do cíngulo anterior (Mason et al., 2007).

As limitações do presente estudo foram: o tamanho da amostra, que foi reduzida pois os indivíduos não apresentavam disponibilidade para comparecerem em três visitas; a disponibilidade da RM, pois a mesma era do Hospital das Clínicas da Unicamp, com difícil agendamento dos testes; encontrar estudos para discussão pois o assunto é inovador e começou a ser pesquisado recentemente.

\section{CONCLUSÃo}

Como observado o $\mathrm{CHO}$ mostrou um importante papel na ativação de áreas cerebrais durante o exercício, ativando áreas relacionadas à tomada de decisões (insula) e motivação (sistema límbico, giro do cíngulo) e desativando principalmente áreas do lobo frontal e precuneos.

Durante todo o estudo, as áreas responsáveis pela iniciação e manutenção dos movimentos, localizadas principalmente no lobo frontal e cerebelo, apresentaram-se ativas. Os resultados entre o consumo do placebo e o momento controle mostraram que a substância placebo não trouxe diferença de ativação neural, sendo que o inverso ocorreu na comparação entre $\mathrm{CHO}$ e controle.

$\mathrm{O} \mathrm{CHO}$ apresentou efeito positivo na ativação de áreas cerebrais quando se comparou a ativação antes e após o consumo, pois se viu que após o consumo, algumas áreas relacionadas a efeitos negativos no exercício não apresentavam ativação após o consumo.

Com isso, a influência do consumo de suplemento de $\mathrm{CHO}$ mostrou-se positiva na atividade cerebral, o que traz novas perspetivas sobre a relação entre o exercício físico, a nutrição e as neurociências, enfatizando o cérebro durante o exercício físico e sua demanda. 
Agradecimentos:

Nada a declarar.

\section{Conflito de Interesses:}

Nada a declarar.

Financiamento:

Nada a declarar

\section{REFERÊNCIAS}

Anantaraman, R., Carmines, A. A., Gaesser, G. A., \& Weltman, A. (1995). Effects of carbohydrate supplementation on performance during 1 hour of high-intensity exercise. Int $J$ Sports Med, 16(7), 461-465.

Bartels, A., \& Zeki, S. (2000). The neural basis of romantic love. Neuroreport, 11(17), 3829-3834.

Benwell, N. M., Mastaglia, F. L., \& Thickbroom, G. W. (2007). Changes in the functional MR signal in motor and non-motor areas during intermittent fatiguing hand exercise. Exp Brain Res, 182(1), 93-97.

Borg, G. (1962). A simple rating scale for use in physical work tests. . Kungl. Fysiografiska Sallskapets I Lund Forhandlinger, 32(2), 7-15.

Bromm, B. (2001). Brain images of pain. News Physiol Sci, 16, 244-249.

Burke, L. M., Hawley, J. A., Wong, S. H., \& Jeukendrup, A. E. (2011). Carbohydrates for training and competition. J Sports Sci, 29(Suppl 1), S17-27.

Buxton, R. B. (2000). Introduction to functional Magnetic Resonance Imaging: principles and techniques. New York: USA: Cambridge University Press.

Calbet, J. A., Radegran, G., Boushel, R., \& Saltin, B. (2009). On the mechanisms that limit oxygen uptake during exercise in acute and chronic hypoxia: role of muscle mass. J Physiol, 587(Pt 2), 477-490.

Carter, J., Jeukendrup, A. E., \& Jones, D. A. (2005). The effect of sweetness on the efficacy of carbohydrate supplementation during exercise in the heat. Can J Appl Physiol, 30(4), 379-391.

Carter, J., Jeukendrup, A. E., Mundel, T., \& Jones, D. A. (2003). Carbohydrate supplementation improves moderate and high-intensity exercise in the heat. Pflugers Arch, 446(2), 211-219.

Cavanna, A. E. (2007). The precuneus and consciousness. CNS Spectr, 12(7), 545-552.

Chambers, E. S., Bridge, M. W., \& Jones, D. A. (2009). Carbohydrate sensing in the human mouth: effects on exercise performance and brain activity. J Physiol, 587(Pt 8), 1779-1794.

Christensen, L. O., Johannsen, P., Sinkjaer, T., Petersen, N., Pyndt, H. S., \& Nielsen, J. B. (2000).
Cerebral activation during bicycle movements in man. Exp Brain Res, 135(1), 66-72.

Craig, A. D. (2002). How do you feel? Interoception: the sense of the physiological condition of the body. Nat Rev Neurosci, 3(8), 655-666.

Craig, A. D. (2009). How do you feel--now? The anterior insula and human awareness. Nat Rev Neurosci, 10(1), 59-70.

Dai, T. H., Liu, J. Z., Sahgal, V., Brown, R. W., \& Yue, G. H. (2001). Relationship between muscle output and functional MRI-measured brain activation. Exp Brain Res, 140(3), 290-300.

Davis, J. M., \& Brown, A. S. (2001). Carboidratos, hormônios e performance em exercícios de resistência. Sports Science Exchange. Gatorade Sports Science Institute, 14(1).

Evans, M., \& Amiel, S. A. (1998). Carbohydrates as a cerebral metabolic fuel. J Pediatr Endocrinol Metab, 11(Suppl 1), 99-102.

Fares, E.-J. M., \& Kayser, B. (2011). Carbohydrate mouth rinse effects on exercise capacity in preand postprandial States. Journal of Nutrition and Metabolism, 2011, 385962. https://doi.org/10.1155/2011/385962

Fontes, E. B., Okano, A. H., De Guio, F., Schabort, E. J., Min, L. L., Basset, F. A., ... Noakes, T. D. (2015). Brain activity and perceived exertion during cycling exercise: an fMRI study. British Journal of Sports Medicine, 49(8), 556-560. https://doi.org/10.1136/bjsports-2012-091924

Franke, W. D., Boettger, C. F., \& McLean, S. P. (2000). Effects of varying central command and muscle mass on the cardiovascular responses to isometric exercise. Clin Physiol, 20(5), 380-387.

Gam, S., Guelfi, K. J., \& Fournier, P. A. (2013). Opposition of carbohydrate in a mouth-rinse solution to the detrimental effect of mouth rinsing during cycling time trials. Int J Sport Nutr Exerc Metab, 23(1), 48-56.

Gant, N., Stinear, C. M., \& Byblow, W. D. (2010). Carbohydrate in the mouth immediately facilitates motor output. Brain Research, 1350, $151-158$. https://doi.org/10.1016/j.brainres.2010.04.004

Heeger, D. J., \& Ress, D. (2002). What does fMRI tell us about neuronal activity? Nat Rev Neurosci, 3(2), 142-151.

Henderson, L. A., Bandler, R., Gandevia, S. C., \& Macefield, V. G. (2006). Distinct forebrain activity patterns during deep versus superficial pain. Pain, 120(3), 286-296.

Hilty, L., Langer, N., Pascual-Marqui, R., Boutellier, U., \& Lutz, K. (2011). Fatigue-induced increase in intracortical communication between mid/anterior insular and motor cortex during cycling exercise. Eur J Neurosci, 34(12), 20352042.

Ide, K., \& Secher, N. H. (2000). Cerebral blood flow and metabolism during exercise. Prog Neurobiol, 61(4), 397-414. 
Jeukendrup, A. E. (2003). Modulation of carbohydrate and fat utilization by diet, exercise and environment. Biochem Soc Trans, 31(Pt 6), 12701273.

Jeukendrup, A. E. (2004). Carbohydrate intake during exercise and performance. Nutrition, 20(7-8), 669-677.

Kupers, R. C., Svensson, P., \& Jensen, T. S. (2004). Central representation of muscle pain and mechanical hyperesthesia in the orofacial region: a positron emission tomography study. Pain, 108(3), 284-293.

Liu, J. Z., Lewandowski, B., Karakasis, C., Yao, B., Siemionow, V., Sahgal, V., \& Yue, G. H. (2007). Shifting of activation center in the brain during muscle fatigue: an explanation of minimal central fatigue? Neurolmage, 35(1), 299-307. https://doi.org/10.1016/j.neuroimage.2006.09.0 50

Maddock, R. J., Garrett, A. S., \& Buonocore, M. H. (2001). Remembering familiar people: the posterior cingulate cortex and autobiographical memory retrieval. Neuroscience, 104(3), 667676.

Mason, M. F., Norton, M. I., Van Horn, J. D., Wegner, D. M., Grafton, S. T., \& Macrae, C. N. (2007). Wandering minds: the default network and stimulus-independent thought. Science, 315(5810), 393-395.

McLachlan, R. S. (2009). A brief review of the anatomy and physiology of the limbic system. Can J Neurol Sci, 36(Suppl 2), S84-87.

Medford, N., \& Critchley, H. D. (2010). Conjoint activity of anterior insular and anterior cingulate cortex: awareness and response. Brain Struct Funct, 214(5-6), 535-549.

Mehta, J. P., Verber, M. D., Wieser, J. A., Schmit, B. D., \& Schindler-Ivens, S. M. (2009). A novel technique for examining human brain activity associated with pedaling using fMRI. J Neurosci Methods, 179(2), 230-239.

Miller, E. K., Freedman, D. J., \& Wallis, J. D. (2002). The prefrontal cortex: categories, concepts and cognition. Philos Trans $R$ Soc Lond B Biol Sci, 357(1424), 1123-1136.

Park, J. Y., Gu, B. M., Kang, D. H., Shin, Y. W., Choi, C. H., Lee, J. M., et al. (2010). Integration of cross-modal emotional information in the human brain: an fMRI study. Cortex, 46(2), 161-169.

Rodriguez, N. R., Di Marco, N. M., \& Langley, S. (2009). American College of Sports Medicine position stand. Nutrition and athletic performance. Med Sci Sports Exerc, 41(3), 709731.

Schneider, S., Brummer, V., Abel, T., Askew, C. D., \& Struder, H. K. (2009). Changes in brain cortical activity measured by EEG are related to individual exercise preferences. Physiol Behav, 98(4), 447452.

Sidhu, S. K., Hoffman, B. W., Cresswell, A. G., \& Carroll, T. J. (2012). Corticospinal contributions to lower limb muscle activity during cycling in humans. J Neurophysiol, 107(1), 306-314.

Thach, W. T. (1998). A role for the cerebellum in learning movement coordination. Neurobiol Learn Mem, 70(1-2), 177-188.

Vissing, J., Andersen, M., \& Diemer, N. H. (1996). Exercise-induced changes in local cerebral glucose utilization in the rat. J Cereb Blood Flow Metab, 16(4), 729-736.

Vogeley, K., Kurthen, M., Falkai, P., \& Maier, W. (1999). Essential functions of the human self model are implemented in the prefrontal cortex. Conscious Cogn, 8(3), 343-363.

Vogt, B. (2009). Cingulate neurobiology and disease: Oxford University Press.

Vogt, B. A., Finch, D. M., \& Olson, C. R. (1992). Functional heterogeneity in cingulate cortex: the anterior executive and posterior evaluative regions. Cereb Cortex, 2(6), 435-443.

Williamson, J. W., McColl, R., Mathews, D., Ginsburg, M., \& Mitchell, J. H. (1999). Activation of the insular cortex is affected by the intensity of exercise. J Appl Physiol, 87(3), 1213-1219. 